\title{
First report on health-related quality of life among children with chronic immune thrombocytopenia in Vietnam
}

\author{
Sang Ngoc Nguyen, $\mathrm{PhD}^{\mathrm{a}, *}$, Nguyen Thi Phuong Thao, MD ${ }^{\mathrm{a}}$, Pham Thi Quynh Van, MD ${ }^{\mathrm{a}}$, \\ Van Dinh Tran, $\mathrm{PhD}^{\mathrm{b}}$
}

${ }^{a}$ Department of Pediatrics, Hai Phong University of Medicine and Pharmacy, Hai Phong, Viet Nam

${ }^{\mathrm{b}}$ Department of Non-Communicable Disease Control and Prevention, National Institute of Hygiene and Epidemiology, Hanoi, Viet Nam

\section{A R T I C L E I N F O}

\section{Keywords:}

Immune thrombocytopenia

ITP

Chronic ITP

Vietnam

\begin{abstract}
A B S T R A C T
Background: Immune thrombocytopenia (ITP) is a benign childhood disease. The aim of this study was to assess Health Related Quality of Life (HRQoL) among chronic ITP children in Vietnam.

Methods: A case - control study was conducted from January 2020 to April 2021. Case group consisted of 56 children with chronic ITP aged from 2 to 18 years and their parents selected in the Department of NephrologyHematology - Endocrinology, Haiphong Children's Hospital, Hai Phong city. Controls included 170 healthy children matched with the patients on sex, and age recruited from schools in the same city. The validated Vietnamese version of the Pediatric Quality of Life Inventory (PedsQL) 4.0 Generic Core Scale was used to assess QoL of the participants.

Results: The mean HRQoL physical, emotional, social, school functioning domains and total scores of the chronic ITP patients were significantly lower compared with that of the controls ( $\mathrm{p}<0.001$ for all). Children in the age group 13-18 had the lowest overall HRQoL score compared other age groups $(60.87 \pm 7.66, p=0.023$ ), especially in the emotional and social functioning domains $(58.33 \pm 6.83 ; 64.17 \pm 11.58$, respectively) $(\mathrm{p}<$ 0.001). Chronic ITP patients with older age, longer treatment duration, and ongoing treatment status showed to be having lower HRQoL scores than the younger one, shorter treatment duration, and the stopped treatment group.

Conclusion: Chronic ITP has a negative impact on HRQoL in terms of physical functioning, emotional, social, school functioning, and overall scores when compared with the healthy sex, age-matched children.
\end{abstract}

\section{Introduction}

Immune thrombocytopenia (ITP) is a common bleeding disorder in blood and hematopoietic diseases. It is characterized by isolated thrombocytopenia (platelet count $<100 * 10^{9} /$ ) due to the unknown existence of autoantibodies in peripheral blood. These autoantibodies are directed against platelet membrane antigens. ${ }^{1}$ Clinical features of ITP disease are emerged with some symptoms such as: spontaneous cutaneous bleeding (petechiae, purpura, or ecchymoses) or mucosal bleeding that involves to nasal passages, buccal, and gingival surfaces to intracranial hemorrhage. ${ }^{2-4}$ The estimated incidence of childhood ITP is about 4-5 per 100.000 person-years. ${ }^{5}$ Severe morbidity and mortality are rare and occur in approximately $0.1 \%-3 \%$ of patients. ${ }^{6,7}$ In more than $70 \%$ of cases, the disease resolves itself within six months, regardless of the treatment given. ${ }^{8}$ Although childhood ITP usually lasts for a few weeks and months, it is estimated that $25-30 \%$ of children develop chronic situation in the world (PLT $<100 * 10^{9} / 1$ and persisted for more than 12 months). ${ }^{9-11}$ One study conducted in Vietnam also found that the proportion of chronic ITP patients among the general ITP was $22.6 \% .^{12}$

ITP can cause fatigue, challenges with daily activities, reduced physical functioning, increased anxiety, and depression. In addition to certain risks of the problem such serious bleeding, patients experience both physical and emotional consequences living with their disease on a daily basis. ${ }^{13}$ Despite benign nature of chronic ITP, treatment and health services expenditures are a burden for families, especially for those with low income or no health insurance. For children, they have to undergo some restrictions in physical activities, disruption in education and social activities because of hospitalization and changes in emotion and psychology over the recurrences of illness. ${ }^{14-16}$

\footnotetext{
* Corresponding author. Department of Pediatrics, Hai Phong University of Medicine and Pharmacy, Hai Phong, Vietnam.

E-mail address: nnsang@hpmu.edu.vn (S.N. Nguyen).
} 
The World Health Organization defines that QoL as "an individual's perception of their position in life in the context of the culture and value systems in which they live and in relation to their goals, expectations, standards and concerns". ${ }^{17}$ This is a wide concept and is influenced by many different factors such as economic status, accommodation, employment, affection, health, ${ }^{18}$ and the level of satisfaction in these areas that people consider the most important in life. When looking insights into the healthcare, people tend to consider the extent of the disease's impact on patient's satisfaction in areas of life and since then, scientists have isolated a 'Health Related Quality of Life' (HRQoL) concept. This is considered an important indicator of the effectiveness of disease treatment. Based on the definition about health of the World Health Organization, the being interested areas of HRQoL are: physical, mental, social and school functioning. The purpose of the assessment of HRQoL in a patient is to determine the extent of the impact of a disease on the life of patients, but not to evaluate the effectiveness or outcome of treatment. $^{19}$

HRQoL is considered a measurement of the health state of an individual and is increasingly becoming an important evaluation tool to evaluate outcome of health improvement interventions and healthcare services. ${ }^{20}$ In recent years, several researchers have concerned and supported HRQoL assessment for patients with chronic diseases. ${ }^{21,22}$ Regarding chronic ITP, although some studies were conducted to assess the HRQoL among the chronic ITP patients, ${ }^{23,24}$ knowledge on HRQoL among the group is still limited. ${ }^{25}$ Literature showed that ITP have negative impact on HRQoL of children. The HRQoL of children with ITP and their parents were remarkably lower in comparison with the healthy children. $^{26,27}$

In Vietnam, research on HRQoL have been paid attention recently, ${ }^{28,29}$ however, HRQoL for chronic ITP patients is still lack of in the country. This study aims to evaluate the HRQoL among Vietnamese children with chronic ITP.

\section{Methods}

\subsection{Patients}

This case-control study was done at the Department of Nephrology Hematology - Endocrinology, Hai Phong Children's Hospital, Hai Phong city, Vietnam from January 2020 to April 2021. The enrolled patients were known recorded chronic ITP at the Department of NephrologyHematology - Endocrinology. The patients or their parents or legal guardians of the patients were interviewed in person by regular visits to pediatric hematology to collect data. Fifty-six patients aged 2-18 years with chronic ITP were selected into this study.

\subsection{Inclusion criteria}

Children aged from 2 to 18 years old diagnosed chronic ITP were selected into the study. Patients were diagnosed with chronic ITP if they had hemorrhagic symptoms such as spontaneous cutaneous or mucosal bleeding or to severe with internal bleeding and isolated thrombocytopenia (platelet count $<100 * 10^{9} / 1$ persisted for over 12 months) via complete blood count or peripheral blood smear.

\subsection{Exclusion criteria}

We excluded from the study: Patients with secondary ITP or symptoms of hepatomegaly, splenomegaly, swollen lymph nodes. Besides, those having disorders relating to physical function or congenital abnormalities (including both children and their parents) were excluded because of difficulties in answering accurately the questionnaire.

\subsection{Control group}

One hundred and seventy healthy age and sex matched students selected during school days were invited to participate in this study as the controls. They were confirmed with no disease history including suffering from acute/chronic diseases or hospitalization in the past one month as well as no diagnosis with intellectual/psychological retardation by trained specialists. Schools were randomly selected based on the urban, rural criteria. In each area one pre-primary school, one primary school, one secondary school, and one high school were randomly chosen.

\subsection{Data collection}

General information of the participants, including age, sex, and residence were gathered. In addition, for the chronic ITP patients, information such as diagnosis age, treatment duration, treatment status and appearance characteristics were also obtained.

The PedsQL 4.0 Generic Core Scales ${ }^{30}$ were utilized to assess the HRQoL of the study participants. The PedsQL 4.0 Generic Core Scales is a generic questionnaire that measures HRQL by a self-report and/or a parent-proxy report for patients aged from 2 to 18 years. ${ }^{30}$ Parent proxy-reports and age-specific child self-reports were similar versions, with formulations appropriate to the understanding of the responder. We used the child version (self-report) for 8-12 years old, the teen version (self-report) for children aged 13-18 years. For young children who aged 2-7 years, we collected the parent's answers since children cannot answer it by themselves. ${ }^{30,31}$

This scale consists of 23 items, scored to assess the difficulty level of children about 4 essential domains: physical functioning (8 items), emotional (5 items), social (5 items) and school functioning (5 items) in the past one month. The difficulty levels are assessed $0-5$ points with frequency of problem: never, rarely, sometimes, often, always, respectively. These raw scores are standardly transformed into scores on a scale from 0 to 100 and calculated following this formula: the mean score is represented by the sum of the items divided by the number of items. ${ }^{30,32}$

This questionnaire was translated into Vietnamese and validated in the Vietnamese context. The validated questionnaire showed to be reliable and valid to assess HRQoL for children in Vietnam. ${ }^{33}$

Researchers were trained carefully about the questionnaire and how to approach the study participants. Pilot data collection was done in both hospital and schools before the actual interviews were performed, making sure the researchers collected the data in the same manner in the two settings. The trained researchers based on the hospital and the schools on the day of interviewing. The participants were explained about the study. After getting the consent form signed, the parents or the patients/students were interviewed in a private room. They were allocated enough time to answer all of the question of the questionnaire.

\subsection{Statistical analysis}

Data were performed and analyzed by SPSS (Statistical Package for the Social Sciences) program version 26.0. Sociodemographic was presented in number, percentage, mean and standard deviation.

The HRQoL scores in each dimension and the total scores were presented with mean and standard deviation. Chi-square test was used to examine the equivalence between the case, and control group. In addition, One-way ANOVA and independent sample T test were used to compare the means between different groups, and the relationship between HRQoL and some characteristics among the patients. The level of significance for all statistical tests was set at $\mathrm{p}<0.05$.

\subsubsection{Ethic}

The research protocol was approved by the Institutional Review Board of Hai Phong University of Medicine and Pharmacy (264/QĐYDHP dated February 5th, 2021). The study was explained in detail to the participant children and their parents or legal guardians after assuring confidentiality of the data. 


\section{Results}

Table 1 shows the socio-demographic details of study groups. No significant differences in age mean, age groups, gender and residence between the two groups ( $p>0.05$ ). More than $60 \%$ in both groups are male. Almost three quarters of participants in both groups reside in rural areas.

In terms of 56 chronic ITP patients, their diagnosis age was $4.37 \pm$ 2.55 years. Most of them treated from 1 to 5 years (67.9\%). There were $43(76.8 \%)$ chronic ITP children were on treatment.

The mean scores of HRQoL were presented in Table 2. The HRQoL scores in five domains of the chronic ITP group were significant lower than that of the controls $(\mathrm{p}<0.001)$. In general, the overall score of HRQoL among the patients and the controls were 68.10, and 91.12, respectively, $\mathrm{p}<0.001$. For all groups, school functioning was the domain with lowest scores while the emotional functioning showed to be the highest score of HRQoL.

The HRQoL in the chronic ITP and control groups classified by age groups, gender, and residence were reported in Table 3. For all subgroups, higher score were found in the control group in comparison to the patient group $(\mathrm{p}<0.001)$.

Table 4 illustrates the comparison of the HRQoL scores in all domains of the patient group based on some characteristics. There were significant differences in the scores based on age group, sex, treatment duration, and treatment status. For age groups, older group showed to be having lower overall score $(\mathrm{p}=0.010)$. Males had higher scores than that of females in all domains. For the treatment duration, the longer treatment the lower HRQoL score reported ( $p<0.05$ except school functioning domain). Patients stopped treatment showed significant higher HRQoL scores in almost all domains than that of patients who were undergoing treatment.

\section{Discussion}

To the best of our knowledge, this is the first study that was conducted to measure the HRQoL among chronic ITP children in Vietnam after a comprehensive review. Our research encompasses 56 patients diagnosed with chronic ITP and 170 healthy children matched with age, gender. The study reported that chronic ITP patients had significant lower HRQoL scores compared with that of the healthy children $(\mathrm{p}<$

Table 1

Characteristics of the chronic ITP group and control group.

\begin{tabular}{|c|c|c|c|}
\hline Variables & $\begin{array}{l}\text { Chronic ITP group } \mathrm{n} \\
(\%)\end{array}$ & $\begin{array}{l}\text { Control group n } \\
(\%)\end{array}$ & $\mathrm{p}$ \\
\hline Age (Mean \pm SD) & $7.79 \pm 3.63$ & $8.41 \pm 3.86$ & 0.277 \\
\hline \multicolumn{4}{|l|}{ Age groups } \\
\hline $2-7$ & $28(50.0 \%)$ & $36(49.9 \%)$ & \multirow[t]{3}{*}{0.994} \\
\hline $8-12$ & $22(39.3 \%)$ & $67(39.4 \%)$ & \\
\hline $13-18$ & $6(10.7 \%)$ & $19(11.2 \%)$ & \\
\hline \multicolumn{4}{|l|}{ Gender } \\
\hline Males & $34(60.7 \%)$ & $103(60.6 \%)$ & \multirow[t]{2}{*}{0.987} \\
\hline Females & $22(39.3 \%)$ & $67(39.4 \%)$ & \\
\hline \multicolumn{4}{|l|}{ Residence } \\
\hline Urban & $14(25 \%)$ & $45(26.5 \%)$ & \multirow[t]{3}{*}{0.828} \\
\hline Rural & $42(75 \%)$ & $125(73.5 \%)$ & \\
\hline $\begin{array}{l}\text { Diagnosis age (Mean } \pm \\
\text { SD) }\end{array}$ & $4.37 \pm 2.55$ & & \\
\hline \multicolumn{4}{|l|}{ Treatment duration } \\
\hline$<1$ year & $11(19.6 \%)$ & & \\
\hline $1-5$ years & $38(67.9 \%)$ & & \\
\hline$>5$ years & $7(12.5 \%)$ & & \\
\hline \multicolumn{4}{|l|}{ Treatment status } \\
\hline On treatment & $43(76.8 \%)$ & & \\
\hline Stopped & $13(23.2 \%)$ & & \\
\hline \multicolumn{4}{|l|}{ Appearance characteristics } \\
\hline Cushing & $30(53.6 \%)$ & & \\
\hline Hirsutism & $19(33.9 \%)$ & & \\
\hline Acne & $5(8.9 \%)$ & & \\
\hline
\end{tabular}

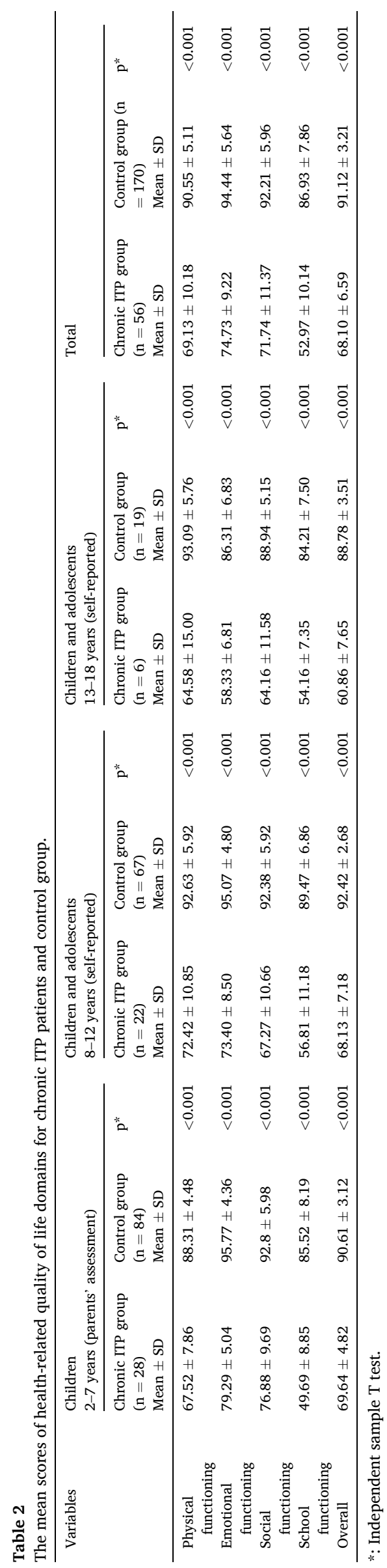


Table 3

Comparison of the overall health-related quality of life score between the chronic ITP group and control groups based on some characteristics.

\begin{tabular}{llll}
\hline Parameters & Chronic ITP group Mean \pm SD & $\begin{array}{l}\text { Control group } \\
\text { Mean } \pm \text { SD }\end{array}$ & $\mathrm{p}^{*}$ \\
\hline Age groups & & & \\
$2-7$ & $69.64 \pm 4.82$ & $90.61 \pm 3.12$ & $<0.001$ \\
$8-12$ & $68.13 \pm 7.18$ & $92.42 \pm 2.68$ & $<0.001$ \\
$\quad 13-18$ & $60.86 \pm 7.65$ & $88.78 \pm 3.51$ & $<0.001$ \\
Gender & & & \\
Males & $69.54 \pm 6.01$ & $90.78 \pm 3.06$ & $<0.001$ \\
Females & $65.89 \pm 6.95$ & $91.65 \pm 3.39$ & $<0.001$ \\
Residence & & & \\
Urban & $69.17 \pm 7.03$ & $91.31 \pm 3.07$ & $<0.001$ \\
Rural & $67.75 \pm 6.48$ & $91.05 \pm 3.28$ & $<0.001$ \\
\hline
\end{tabular}

*: Independent sample T test or One-way ANOVA.

0.05). The research also found that chronic ITP patients having certain characteristics, including older age, longer duration of treatment, and undergoing treatment, had lower HRQoL scores than their counterparts.

In present study, the HRQoL of chronic ITP patients was significantly lower than that of healthy children for all domains, regardless of age, gender or residence characteristics. This result was in line with previous studies, ${ }^{27,34}$ but contrary with a study conducted in Dutch population which found no significant difference in HRQoL between newly diagnosed ITP patients and the reference group, ${ }^{16}$ both using the PedsQL questionnaires. The latter difference could be explained by the tools using to assess the HRQoL of that study and the current one.

Parental assessment may relate to the fear of bleeding and injury, as well as the uncertainty about their children's future. Meanwhile, for the patients who are teenagers and adolescents with a certain understanding of their conditions, their priorities might relate to the fear of interacting with people's surroundings, especially their peers. Study in China also showed that parents are much more concern with the disease than their children, resulting seriously influence their HRQoL. ${ }^{27}$

Results from the current study also demonstrates significant differences between the HRQoL scores and some demographic and clinical characteristics. Children aged 13-18 years had lowest HRQoL overall and specifically in emotional and social functioning, while children in the age group 2-7 years had impaired HRQoL in school functioning. As mentioned above, these findings could be interpreted by the different priorities related to the age group of children and parents. In terms of clinical characteristics, the patients being on treatment or having been treated for more than 5 years had lower HRQoL scores than those who stopped treatment or had treatment duration less than 5 years. This was predictable since previous studies revealed that children with persistent ITP had worse HRQoL. ${ }^{27,35}$ The reason for this could be attributed to the burden of prolonged treatment that the parents have to endure and leads to restriction of their child's daily activities, which might reduce the child's HRQoL. Besides, the long-term treatment with corticoids could also worsen HRQoL in children. ${ }^{36}$ However, some studies found improvements in HRQoL of children with chronic ITP versus newly diagnosed patients, suggesting other possible aspects that could influence the HRQoL in addition to the time of treatment course. For the relationship between the HRQoL scores and gender, we found the worse overall HRQoL in females. Although HRQoL tends to decrease in females, the selection bias in assessing the HRQoL scores should also be taken into consideration. Parents' perception and/or the process of expressing emotions or socializing with their peers might be influenced by gender factor rather than the real ability of the children.

Given the negative impact of the problem on children, intervention program emphasizing spiritual growth, physical activity, and communications are necessary for children with chronic ITP. Additionally, different health issues such as pain, anxiety, fatigue have been reported by chronic ITP children. ${ }^{13}$ Those issues may have affect on quality of life among those patients. Future studies need to be conducted to measure the effect of those aspects on patients living with chronic ITP.

Some limitations of the current study need to be consideration. First, a relatively small sample size did not allow us to analyze clinically relevant subgroups such as acute ITP. In addition, the case of the present study was only selected in one hospital a city in the North of Vietnam. Second, although PedSQL 4.0 is an international tool to measure HRQL, it evaluates the HRQoL for children with general health, not particular ITP. Taking into account these limitations, it would be interesting to study the quality of life of the ITP patients with a larger cohort with different tools will be applied.

Table 4

Comparison between different health-related quality of life scores regarding demographic and clinical characteristics in the chronic ITP group.

\begin{tabular}{|c|c|c|c|c|c|c|c|c|c|c|}
\hline \multirow[t]{2}{*}{ Parameters } & \multicolumn{2}{|c|}{ Physical functioning } & \multicolumn{2}{|c|}{ Emotional functioning } & \multicolumn{2}{|c|}{ Social functioning } & \multicolumn{2}{|c|}{ School functioning } & \multicolumn{2}{|l|}{ Overall } \\
\hline & Mean \pm SD & $\mathrm{p}^{*}$ & Mean \pm SD & $\mathrm{p}^{*}$ & Mean \pm SD & $\mathrm{p}^{*}$ & Mean \pm SD & $\mathrm{p}^{*}$ & Mean \pm SD & $\mathrm{p}^{*}$ \\
\hline \multicolumn{11}{|l|}{ Age groups } \\
\hline $2-7$ & $67.52 \pm 7.86$ & 0.122 & $79.28 \pm 5.03$ & $<0.001$ & $76.87 \pm 9.68$ & 0.002 & $49.68 \pm 8.85$ & 0.042 & $69.63 \pm 4.82$ & 0.010 \\
\hline $8-12$ & $72.42 \pm 10.85$ & & $73.41 \pm 8.51$ & & $67.27 \pm 10.66$ & & $56.82 \pm 11.19$ & & $68.13 \pm 7.19$ & \\
\hline $13-18$ & $64.58 \pm 15$ & & $58.33 \pm 6.83$ & & $64.17 \pm 11.58$ & & $54.17 \pm 7.36$ & & $60.87 \pm 7.66$ & \\
\hline \multicolumn{11}{|l|}{ Sex } \\
\hline Males & $70.79 \pm 10.69$ & 0.132 & $75.44 \pm 8.91$ & 0.479 & $73.46 \pm 9.96$ & 0.163 & $54.41 \pm 10.03$ & 0.188 & $69.54 \pm 6.01$ & 0.041 \\
\hline Females & $66.58 \pm 9.52$ & & $73.64 \pm 9.78$ & & $69.09 \pm 13.06$ & & $50.74 \pm 10.12$ & & $65.89 \pm 6.95$ & \\
\hline \multicolumn{11}{|l|}{ Residence } \\
\hline Urban & $69.48 \pm 10.83$ & 0.883 & $73.57 \pm 7.70$ & 0.591 & $74.82 \pm 10.58$ & 0.245 & $56.43 \pm 10.46$ & 0.142 & $69.18 \pm 7.03$ & 0.487 \\
\hline Rural & $69.01 \pm 10.09$ & & $75.12 \pm 9.72$ & & $7071 \pm 11.56$ & & $51.82 \pm 9.88$ & & $67.75 \pm 6.48$ & \\
\hline \multicolumn{11}{|l|}{ Treatment duration } \\
\hline$<1$ year & $67.45 \pm 8.19$ & 0.047 & $79.09 \pm 5.39$ & $<0.001$ & $81.36 \pm 7.01$ & $<0.001$ & $50.27 \pm 11.71$ & 0.623 & $70.74 \pm 4.94$ & $<0.001$ \\
\hline $1-5$ years & $71.09 \pm 9.56$ & & $76.05 \pm 8.07$ & & $70.86 \pm 10.30$ & & $53.64 \pm 9.99$ & & $68.93 \pm 5.51$ & \\
\hline$>5$ years & $61.16 \pm 13.12$ & & $60.71 \pm 7.32$ & & $61.43 \pm 12.15$ & & $53.57 \pm 9.0$ & & $59.47 \pm 8.08$ & \\
\hline \multicolumn{11}{|l|}{ Treatment status } \\
\hline On treatment & $67.71 \pm 9.95$ & 0.057 & $73.95 \pm 9.17$ & 0.254 & $70.29 \pm 11.13$ & 0.082 & $51.67 \pm 10.82$ & 0.021 & $66.84 \pm 6.3$ & 0.008 \\
\hline Stopped & $73.83 \pm 9.89$ & & $77.31 \pm 9.27$ & & $76.54 \pm 11.25$ & & $57.28 \pm 5.92$ & & $72.29 \pm 5.92$ & \\
\hline \multicolumn{11}{|c|}{ Appearance characteristics } \\
\hline Having Cushing & $68.11 \pm 10.49$ & 0.425 & $74.50 \pm 11.01$ & 0.837 & $69.25 \pm 12.13$ & 0.078 & $52.26 \pm 10.81$ & 0.581 & $66.73 \pm 7.6$ & 0.094 \\
\hline No Cushing & $70.31 \pm 9.94$ & & $75 \pm 6.78$ & & $74.62 \pm 9.89$ & & $53.78 \pm 9.45$ & & $69.69 \pm 4.83$ & \\
\hline Having Hirsutism & $71.52 \pm 11.21$ & 0.212 & $71.84 \pm 11.33$ & 0.142 & $67.37 \pm 11.59$ & 0.038 & $53.51 \pm 11.45$ & 0.778 & $66.89 \pm 8.36$ & 0.325 \\
\hline No Hirsutism & $67.91 \pm 9.57$ & & $76.22 \pm 7.67$ & & $73.99 \pm 10.73$ & & $52.69 \pm 9.55$ & & $68.73 \pm 5.47$ & \\
\hline Having Ance & $70.0 \pm 17.48$ & 0.91 & $61.0 \pm 6.52$ & $<0.001$ & $69.0 \pm 6.52$ & 0.577 & $57.0 \pm 6.71$ & 0.356 & $65.0 \pm 6.85$ & 0.272 \\
\hline No Acne & $69.05 \pm 9.48$ & & $76.08 \pm 8.33$ & & $72.0 \pm 11.75$ & & $52.57 \pm 10.37$ & & $68.41 \pm 6.54$ & \\
\hline
\end{tabular}

*: Independent sample T test or One-way ANOVA. 


\section{Conclusion}

Chronic ITP has a negative impact on HRQoL in terms of physical functioning, emotional, social, school functioning, and overall scores when compared with the healthy sex, age-matched children. Patients with older age, longer treatment duration, and ongoing treatment status showed to be having lower HRQoL scores than the younger one, shorter treatment duration, and the stopped treatment group.

\section{Funding}

The study received no funding.

\section{Declaration of competing interest}

No conflict of interest is declared.

\section{Acknowledgement}

We would like to sincere thanks to the children and their parents/ caregivers who participated in this study. Thanks also due to the Hai Phong Children's Hospital, and schools in Hai Phong city which supported us during the data collection.

\section{References}

1 Bakchoul T, Sachs UJ. Platelet destruction in immune thrombocytopenia. Understanding the mechanisms. Hämostaseologie. 2016;36(3):187-194.

2 Kühne T, Berchtold W, Michaels LA, et al. Newly diagnosed immune thrombocytopenia in children and adults: a comparative prospective observational registry of the Intercontinental Cooperative Immune Thrombocytopenia Study Group. Haematologica. 2011;96(12):1831-1837.

3 Revel-Vilk S, Yacobovich J, Frank S, et al. Age and duration of bleeding symptoms at diagnosis best predict resolution of childhood immune thrombocytopenia at 3,6 , and 12 months. J Pediatr. 2013;163(5):1335-1339. e1-1339.

4 Neunert C, Noroozi N, Norman G, et al. Severe bleeding events in adults and children with primary immune thrombocytopenia: a systematic review. J Thromb Haemostasis. 2015;13(3):457-464.

5 Yong M, Schoonen WM, Li L, et al. Epidemiology of paediatric immune thrombocytopenia in the general practice research database. Br J Haematol. 2010; 149(6):855-864.

6 Butros LJ, Bussel JB. Intracranial hemorrhage in immune thrombocytopenic purpura: a retrospective analysis. J Pediatr Hematol Oncol. 2003;25(8):660-664.

7 Neunert CE, Buchanan GR, Imbach P, et al. Severe hemorrhage in children with newly diagnosed immune thrombocytopenic purpura. Blood. 2008;112(10): 4003-4008.

8 Rodeghiero F. Idiopathic thrombocytopenic purpura: an old disease revisited in the era of evidence-based medicine. Haematologica. 2003;88(10):1081-1087.

9 Blanchette V, Bolton-Maggs P. Childhood immune thrombocytopenic purpura: diagnosis and management. Hematol. Oncol. Clin. N. Am. 2010;24(1):249-273.

10 Demircioğlu F, Saygi M, Yilmaz S, et al. Clinical features, treatment responses, and outcome of children with idiopathic thrombocytopenic purpura. Pediatr Hematol Oncol. 2009;26(7):526-532.

11 Imbach P, Kühne T, Müller D, et al. Childhood ITP: 12 months follow-up data from the prospective registry I of the intercontinental childhood ITP study group (ICIS). Pediatr Blood Cancer. 2006;46(3):351-356.

12 Nguyen Thi Mong Hong, Hieu LTM, Tuan DA, et al. Characteristics of children chronic immune thrombocytopenic purpura at Children's Hospital 1 from 7/2009 to 6/2010. Ho Chi Minh City: Children's Hospital 1. Ho Chi Minh City; 2011.
13 Kruse C, Kruse A, DiRaimo J. Immune thrombocytopenia: the patient's perspective. Ann. Blood. 2020;6.

14 Zhang H, Wang L, Quan M, et al. Health-related quality of life in children with chronic immune thrombocytopenia in China. Health Qual Life Outcome. 2016;14:45.

15 Neunert CE, Buchanan GR, Blanchette V, et al. Relationships among bleeding severity, health-related quality of life, and platelet count in children with immune thrombocytopenic purpura. Pediatr Blood Cancer. 2009;53(4):652-654.

16 Heitink-Pollé KM, Haverman L, Annink KV, et al. Health-related quality of life in children with newly diagnosed immune thrombocytopenia. Haematologica. 2014;99 (9):1525-1531.

17 World Health Organization. Programme on Mental Health WHOQOL User Manual. Geneva: World Health Organization; 1998.

18 Burckhardt CS, Anderson KL. The quality of life scale (QOLS): reliability, validity, and utilization. Health Qual Life Outcome. 2003;1(1):60.

19 Neunert C, Lim W, Crowther M, et al. The American Society of Hematology 2011 evidence-based practice guideline for immune thrombocytopenia. Blood. 2011;117 (16):4190-4207.

20 Varni JW, Burwinkle TM, Lane MM. Health-related quality of life measurement in pediatric clinical practice: an appraisal and precept for future research and application. Health Qual Life Outcome. 2005;3:34.

21 D'Souza MS, O'Mahony J, Karkada SN. Effectiveness and meaningfulness of breast cancer survivorship and peer support for improving the quality of life of immigrant women: a mixed methods systematic review protocol. Clin. Epidemiol. Glob. Health. 2021;10:100678.

22 Ibrahim S, Manu MK, James BS, et al. Health related quality of life among patients with chronic obstructive pulmonary disease at a tertiary care teaching hospital in southern India. Clin. Epidemiol. Glob. Health. 2021;10:100711.

23 Mathias SD, Gao SK, Miller KL, et al. Impact of chronic Immune Thrombocytopenic Purpura (ITP) on health-related quality of life: a conceptual model starting with the patient perspective. Health. Qual. Life. Outcomes. 2008;6:13.

24 McMillan R, Bussel JB, George JN, et al. Self-reported health-related quality of life in adults with chronic immune thrombocytopenic purpura. Am J Hematol. 2008;83(2): $150-154$.

25 Grainger JD, Blanchette VS, Grotzinger KM, et al. Health-related quality of life in children with chronic immune thrombocytopenia treated with eltrombopag in the PETIT study. Br J Haematol. 2019;185(1):102-106.

26 Zhou M, Li X-j, Shu H-y, et al. Analysis of the quality of life in children and their parents with immune thrombocytopenia in China. Blood. 2014;124(21):5017.

27 Zhang H, Wang L, Quan M, et al. Health-related quality of life in children with chronic immune thrombocytopenia in China. Health. Qual. Life. Outcomes. 2016;14 (1):45.

28 Tran TH, Trinh NL, Hoang Y, et al. Health-related quality of life among Vietnamese breast cancer women. Cancer Cont. 2019;26(1), 1073274819862787.

29 Nguyen SN, Tran VQ, Vu QV. Quality of life of children with idiopathic nephrotic syndrome according to clinical types. Vietnam. J. Sci. Tech. Engine. 2018;60(1):4.

30 Varni JW, Seid M, Kurtin PS. PedsQL 4.0: reliability and validity of the Pediatric Quality of Life Inventory version 4.0 generic core scales in healthy and patient populations. Med. Care. 2001;39(8):800-812

31 Gheissari A, Farajzadegan Z, Heidary M, et al. Validation of Persian version of PedsQLTM 4.0 ${ }^{\mathrm{TM}}$ generic core scales in toddlers and children. Int J Prev Med. 2012;3 (5):341-350.

32 Varni JW, Burwinkle TM, Seid M, et al. The PedsQL 4.0 as a pediatric population health measure: feasibility, reliability, and validity. Ambul Pediatr. 2003;3(6): 329-341.

33 Trang D, Ha N, Ha L. Validation of Vietnamese version of Pediatric Quality of Life Inventory version 4.0 generic score scale among school children. Southeast Asian J Trop Med Publ Health. 2019;50(5):942-951.

34 Yahia S, Wahba Y, El-Gilany A-H, et al. Psychiatric disorders and quality of life in Egyptian patients with chronic immune thrombocytopenic purpura: a single center study. Ind. J. Hematol. Blood Transfus. 2019;35(2):347-351.

35 Mokhtar GM, Farid SM, Shaker NM, et al. Health-related quality of life of Egyptian children with immune thrombocytopenia and their parents. J Pediatr Hematol Oncol. 2014;36(3):194-199.

36 Provan D, Arnold DM, Bussel JB, et al. Updated international consensus report on the investigation and management of primary immune thrombocytopenia. Blood Adva. 2019;3(22):3780-3817. 